\title{
Thermodynamic properties of ionic fluids over wide ranges of temperature
}

\author{
Kenneth S. Pitzer \\ Department of Chemistry and Lawrence Berkeley Laboratory, \\ University of California, Berkeley, California 94720, U.S.A. \\ Abstract - The principle of corresponding states is valid in reasonable \\ approximation for ionic fluids but the quantitative behavior differs \\ considerably from that for neutral-molecule fluids. The role of a \\ dielectric is such as to make the $\varepsilon \mathrm{T}$ product the effective temperature. \\ Thus aqueous electrolytes near room temperature have very high reduced \\ temperatures on a corresponding states basis. The status of knowledge \\ for various aqueous solutes is summarized. The critical region for an \\ ionic system is difficult to treat theoretically and is out-of-range \\ experimentally for simple substances such as $\mathrm{NaCl}$. Current best approxi- \\ mations are discussed. A model, two-component ionic system with an \\ experimentally convenient critical temperature has been found and its \\ properties investigated. The role of long-range fluctuations on \\ properties in the critical region is considered for ionic systems. Fused \\ salts (without solvent) have low reduced temperatures and they follow \\ corresponding states quite well as has been shown by Reiss and others. \\ Fused salts have a remarkably large coefficient of expansion; its cause \\ is discussed. At low reduced temperature an ionic-particle vapor is \\ largely associated to neutral clusters -- pairs, quartets, etc. An \\ interesting system of considerable engineering and geological importance \\ is $\mathrm{NaCl}$ in steam which has been treated in terms of successive hydration \\ equilibria for the ion-pair.
}

\section{INTRODUCTION}

There are many interesting differences between ionic and nonionic fluids. Those related to dilute aqueous electrolytes were of primary interest to physical chemists about six decades ago and were explained by Debye and Hückel. But there are other ranges of concentration and effective temperature which have only recently been explored where there are also distinct and interesting properties of ionic fluids.

The concept of corresponding states is useful for ionic systems in providing a semi-quantitative correlation of properties in terms of reduced temperature and volume. The essential requirement for corresponding states behavior is similarity of shape of the interparticle potential subject to both energy and distance scaling factors (ref. 1). For the ionic case the similarity of shape is clear; it is the $\mathrm{r}^{-1}$ dependence of the electrostatic potential combined with a sudden repulsive potential. But the ionic case also involves the change in sign for the potential between like-charged particles as compared to that between particles of opposite sign of charge. Reiss et al. (ref. 2) noted these aspects and compared fused salt properties on a corresponding states basis. Subsequently Blander (ref. 3) extended the dimensional theory, while Friedman and Larsen (ref. 4) have considered ionic fluid properties very generally from a corresponding states viewpoint.

If we limit our consideration to symmetrical electrolytes and assume a hard-core interparticle distance a then the energy scaling factor is $\mathrm{Z}^{2} \mathrm{e}^{2} /\left(4 \pi \varepsilon_{0} \varepsilon a\right)$ while the distance factor is $\mathrm{a}$ and the volume factor $\mathrm{a}^{3}$. Here $\mathrm{Z}$ is the number of charges on an ion and $\varepsilon$ is the relative permittivity or dielectric constant. The permittivity of free space is $\varepsilon_{0}$. Much of the literature on electrolytes is written in e.s.u. whereupon the factor $4 \pi \varepsilon_{0}$ is omitted; also the dielectric constant is often represented by D. The reduced variables are $\mathrm{T}_{\mathrm{r}}=\mathrm{T} / \mathrm{T}_{\mathrm{c}}$ and $\mathrm{V}_{\mathrm{r}}=\mathrm{V} / \mathrm{V}_{\mathrm{c}}$. For comparison of different systems, the
above relationships indicate that

and

$$
\begin{aligned}
& \mathrm{T}_{\mathrm{c}}{ }^{\prime \prime}=\mathrm{T}_{\mathrm{c}}{ }^{\prime}\left(\mathrm{Z}^{\prime \prime} / \mathrm{Z}^{\prime}\right)^{2}\left(\varepsilon^{\prime} \mathrm{a}^{\prime} / \varepsilon^{\prime \prime} \mathrm{a}^{\prime \prime}\right) \\
& \mathrm{V}_{\mathrm{c}}{ }^{\prime \prime}=\mathrm{V}_{\mathrm{c}}{ }^{\prime}\left(\mathrm{a}^{\prime \prime} / \mathrm{a}^{\prime}\right)^{3} .
\end{aligned}
$$

From the temperature relationship we note that if $\mathrm{Z}$ and a remain the $\mathrm{same}^{\prime \prime} \varepsilon^{\prime \prime}=\mathrm{T}^{\prime} \varepsilon^{\prime}$, in other words the effective temperature is the dielectric constant-temperature product. With the large value $\varepsilon \cong 80$ for water near room temperature, singly charged aqueous electrolytes are at a very high reduced temperature. 


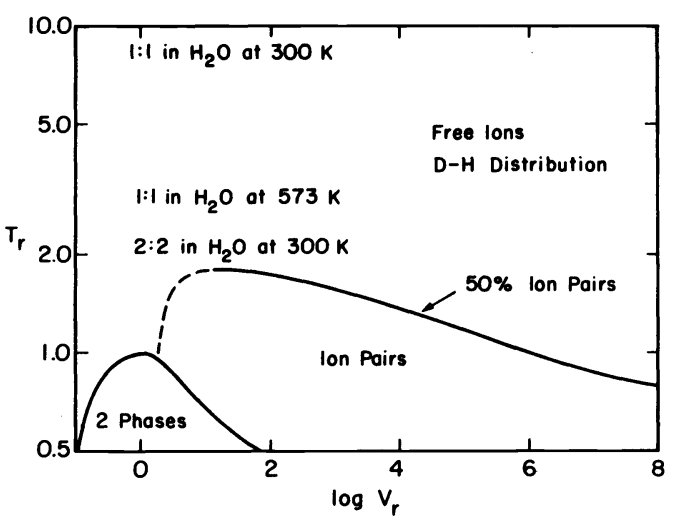

Fig. 1. The phase boundaries and critical point for an ionic fluid ( $\mathrm{NaCl}$ ) on a corresponding states basis. Also shown are the region of ion pairing in the vapor and the approximate reduced temperatures for aqueous electrolytes.

Figure 1 gives an overview based on the properties of pure NaCl for which statistically guided extrapolations of experimental data to higher temperatures (refs. 5,6) yield $\mathrm{T}_{\mathrm{c}} \cong 3900 \mathrm{~K}, \mathrm{~V}_{\mathrm{c}} \cong 490 \mathrm{~cm}^{3} \cdot \mathrm{mol}^{-1}$. The approximate reduced temperatures for $1: 1$ aqueous electrolytes at $300 \mathrm{~K}$ and $573 \mathrm{~K}$ and for $2: 2$ aqueous electrolytes at $300 \mathrm{~K}$ are shown on Fig. 1 . Also indicated is the curve for $50 \%$ ion pairing for $\mathrm{NaC} 1$ vapor.

In the following sections, recent advances for various ranges of reduced temperature and reduced volume will be reported.

\section{AQUEOUS ELECTROLYTES AT HIGH TEMPERATURE}

While there is an extensive data base concerning aqueous electrolytes at or near $298 \mathrm{~K}$, it is only recently that there have been substantial studies at temperatures ranging above $473 \mathrm{~K}$ (refs. 7-11). Since $\varepsilon$ decreases faster than $T$ increases, the effective temperature $\varepsilon T$ is lower at higher T. There are semi-empirical equations that very successfully represent experimental thermodynamic data for mixed as well as pure electrolytes at $298 \mathrm{~K}$ (refs. 12,13) and are currently being tested as measurements become available at high temperature (ref. 15). Once the parameters have been determined, these equations will yield predictions of activity coefficients for mixed electrolytes at various temperatures. I will discuss solubilities of salts in mixed electrolytes at the Second IUPAC Symposium on Solubility Phenomena later this summer, and the paper will appear in this Journal. Hence, this type of ionic fluid will not be considered further here.

\section{CRITICAL REGION: FLUCTUATION EFFECTS}

Particularly interesting are the properties of an ionic systems in the critical region. Near the critical point the gradient of the chemical potential with density or composition decreases to zero and fluctuations of density for single-component systems or of composition for two-component systems can become important. It is known that these fluctuations affect the shape of the two-phase equilibrium curve for neutral-molecule fluids. This is usually discussed in terms of the exponent $\beta$ in the expressions

$$
\begin{aligned}
& \left(\rho^{\prime \prime}-\rho^{\prime}\right)=\mathrm{B}\left[\left(\mathrm{T}_{\mathrm{c}}-\mathrm{T}\right) / \mathrm{T}_{\mathrm{c}}\right]^{\beta} \\
& \left(\mathrm{x}^{\prime \prime}-\mathrm{x}^{\prime}\right)=\mathrm{B}\left[\left(\mathrm{T}_{\mathrm{c}}-\mathrm{T}\right) / \mathrm{T}_{\mathrm{c}}\right]^{\beta}
\end{aligned}
$$

where $\rho^{\prime \prime}-\rho^{\prime}$ is the difference in density between liquid and vapor or $x^{\prime \prime}-x^{\prime}$ is the difference in mole fraction between the more and less concentrated phases at equilibrium. $B$ and $\beta$ are constants and these simple expressions become valid in the limit as the critical point is approached. It is the exponent $\beta$ which is of general significance. $B$ is specific to the particular system, although B-values for one-component systems should follow corresponding states principles where appropriate.

Any reasonable analytic equation of state will yield 0.50 for $\beta$ whereas the observed values for neutral-molecule fluids are in the range 0.30 to 0.35 . There is now an extensive theory of the long-range fluctuations which yields the smaller exponent. The basic concept is that the dimension of the fluctuations exceeds that of the interparticle forces for a substantial region near the critical point.

The $\mathrm{r}^{-1}$ potential between ions, however, has a much longer range than the $\mathrm{r}^{-6}$ potential between neutral molecules. Hence, this fluctuation effect is expected to be much smaller and possibly negligible for ionic systems. An early study of this question is that of Buback and Franck (ref. 15) on $\mathrm{NH}_{4} \mathrm{Cl}$. The phase equilibrium curve fits the "classical" value of $\beta=0.50$ to within a reduced temperature difference of 0.012 . 


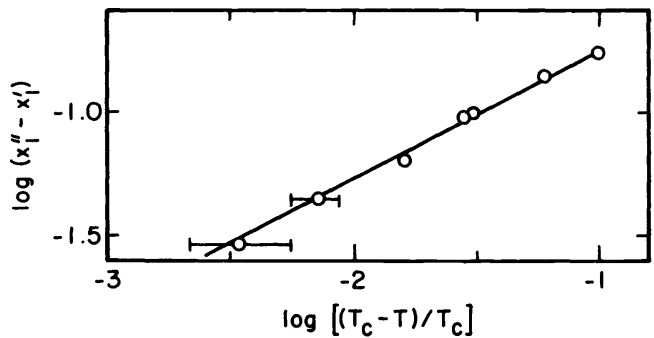

Fig. 2. Logarithmic plot to show the critical exponent $\beta$ for the system tetra-n-butylammonium picrate: 1-chloroheptane. The line shows $\beta=0.50$.

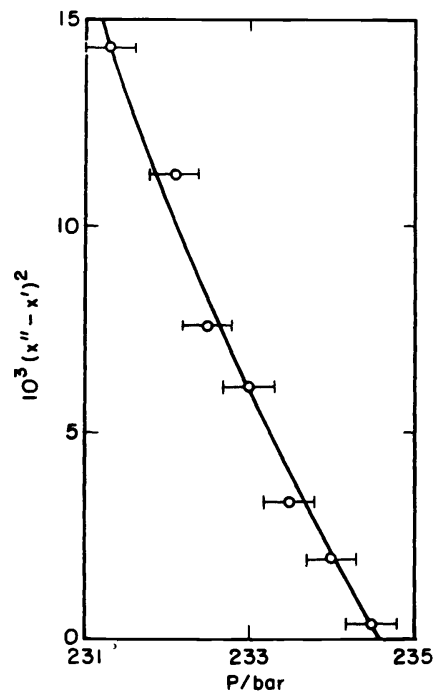

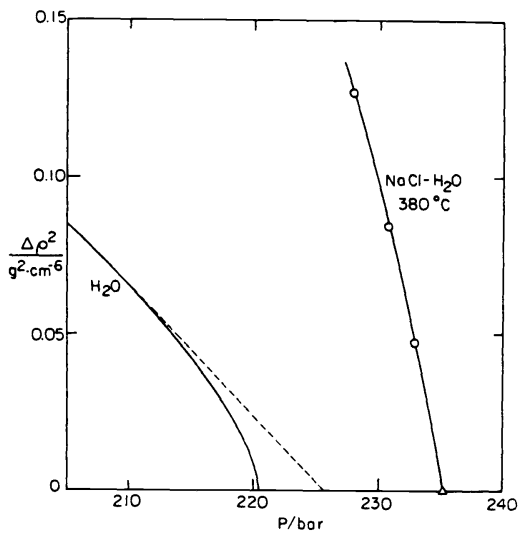

Fig. 3. Estimation of the large-scale fluctuation effect; see equation (5) and the text.
Fig. 4. Test of the large-scale fluctuation effect for $\mathrm{NaCl}-\mathrm{H}_{2} \mathrm{O}$ at $380^{\circ} \mathrm{C}$; see equation (7).

Fig. 5. Conductance near the critical temperature for tetra-n-butylammonium picrate: 1-chloroheptane. The critical composition is indicated.

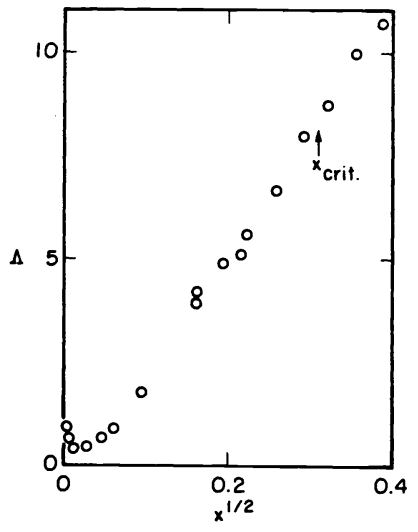

Tetra-n-butylammonium picrate is a well-investigated fused salt melting at $91^{\circ} \mathrm{C}$. We found that its mixture with 1 -chloroheptane shows a critical point at $141.2^{\circ} \mathrm{C}(414.4 \mathrm{~K})$ and mole fraction 0.08 picrate (ref. 16). Figure 2 shows the determination of the critical exponent with the line corresponding to $\beta=0.50$. Evidently the measurements are consistent with this value down to a reduced temperature difference of only 0.003 .

A third ionic critical system is $\mathrm{NaCl}-\mathrm{H}_{2} \mathrm{O}$ for which there are measurements at $380^{\circ} \mathrm{C}$, just above the critical point of pure $\mathrm{H}_{2} \mathrm{O}$ at $374^{\circ} \mathrm{C}$. For isothermal measurements on a system including a volatile component, equations ( 3 and 4 ) may be recast to make pressure the independent variable. Also it is convenient to make additional rearrangements to linearize the dependence on pressure.

$$
\begin{aligned}
& \left(\rho^{\prime \prime}-\rho^{\prime}\right)^{1 / \beta}=B\left(P_{c}-P\right) \\
& \left(x^{\prime \prime}-x^{\prime}\right)^{1 / \beta}=B\left(P_{c}-P\right)
\end{aligned}
$$

Figure 3 shows a plot of the density difference vs the pressure (with $\Delta \rho=\rho^{\prime \prime}-\rho^{\prime}$ ) for both pure water and the $\mathrm{NaCl}-\mathrm{H}_{2} \mathrm{O}$ mixture. The ordinate is $\Delta \rho^{2}$ corresponding to $\beta=0.50$. Thus the data for $\mathrm{NaCl}-\mathrm{H}_{2} \mathrm{O}$ (refs. 17,18) show little curvature and a finite slope and are consistent with $\beta=0.50$. In contrast, the data for pure $\mathrm{H}_{2} \mathrm{O}$ (ref. 19) show substantial curvature and an infinite slope in the limit of $\Delta \rho=0$. Thus $\beta<0.50$ for pure water as is well known. The older measurements of mole fraction for $\mathrm{NaC} 1-\mathrm{H}_{2} \mathrm{O}$ at $380^{\circ} \mathrm{C}$ are not precise enough to be definitive. They suggest a value of $\beta$ larger than 20.50 , but this seems most unlikely. Recently Bischoff and Rosenbauer (ref. 20) have made more precise measurements on the composition of the equilibrium phases of $\mathrm{NaCl}-\mathrm{H}_{2} \mathrm{O}$ at $380^{\circ} \mathrm{C}$ with the results shown on Fig. 4. Here it is apparent that the data indicate a finite slope at $x^{\prime \prime}-x^{\prime}=0$, indicating $\beta=0.50$. Also, the curvature is the opposite of that for pure $\mathrm{H}_{2} \mathrm{O}$ in Fig. 3 . This is consistent with the older data in the characteristic that $\beta$ increases above 0.5 as $x^{\prime \prime}-x^{\prime}$ becomes large.

The general structural picture is clear for both the ionic licuid and the ionic vapor. The liquid has an alternating charge pattern with several ions of the opposite charge around a given central ion. There are no clearly identifiable ion pairs. The electrical conductance is large. 
The saturated vapor of a simple ionic system is very largely ion-paired with substantial further association to neutral quadruples, etc. This is known both from theory for the hard core ionic model (Gillan, ref. 21) and experimentally for various alkali halides, etc. (ref. 22). There is some dissociation to separate ions and there are some charged clusters; hence, the vapor shows some electrical conductance.

$\mathrm{NH}_{4} \mathrm{Cl}$ is an exception to this pattern; its vapor, is a mixture of $\mathrm{HCl}$ and $\mathrm{NH}_{3}$. Since this vapor has much lower chemical potential than the normal ion pairs, quadruples, etc., the critical temperature of $\mathrm{NH}_{4} \mathrm{Cl}$ is much lower than that of an alkali halide with the same radii ( $\mathrm{KC} 1$ ) and other critical properties are also affected. It is known that the electrical conductance of liquid $\mathrm{NH}_{4} \mathrm{Cl}$ remains high to within $30^{\circ} \mathrm{C}$ of the critical point; hence, this liquid appears to be a normal fused salt.

We have little definite knowledge of the structure of an ionic fluid at its critical point. The electrical conductance of the system tetra-n-butylammonium picrate: 1-chloroheptane is now being measured at its critical temperature (ref. 23) and the preliminary results are shown on Fig. 5. At very low concentration there is a minimum in conductance with increasing ionization at both lower and higher concentrations. At higher concentration there are triple ions and other charged clusters. This is a well-known phenomenon which has been represented quantitatively for dilute electrolytes at low reduced temperature (refs. 24,25). In the range of the critical composition, the equivalent conductance increases steadily with mole fraction. This arises from a further decrease in the fraction of nonconducting neutral clusters with conversion to an expanded fused-salt pattern as well as to charged clusters. As the concentration increases, the distinctions between various clusters become ambiguous, and the "expanded fused-salt" description seems more appropriate.

\section{FUSED SALTS}

Fused salts, without a solvent and hence with $\varepsilon=1$, have low reduced temperatures. There are extensive reviews and properties have been compared on a corresponding states basis (refs. 2,3,26-28). I wish at this time only to call attention to one marked difference between fused salts and neutral-molecule liquids. This is the thermal expansion which is much larger for a fused salt. Kirshenbaum et al. (ref. 28) showed that the reduced density increases from 1.0 to about 3.0 for simple liquids such as argon as the reduced temperature drops from 1.0 to 0.5 but that the reduced density of fused salts such as NaCl increased from 1.0 to about 6.0 for the same temperature decrease. This rapid increase in reduced density continues for a fused salt down to the melting point.

It is also noteworthy that this large volumetric expansion of an ionic liquid does not involve an increase in the nearest neighbor interionic distance. Indeed this distance decreases slightly from 2.82 to $2.78 \AA$ on melting of NaCl (ref. 29). Rather, the expansion arises from a decrease in number of nearest neighbors from 6 in the solid to 4.0 in liquid $\mathrm{NaCl}$ near its melting point. Thus, fused salts expand by becoming more open structures with low average coordination numbers and substantial vacant space. The underlying cause is the presence of long-range repulsive forces. As has been shown in more detail elsewhere (ref.6), the decrease in attractive interactions is largely compensated by decrease in repulsive terms as the coordination number decreases for an ionic system. Thus the energy cost of this expansion is relatively small and is overcome by the entropy increase arising from the less restricted motion in the low-coordination-number structure.

\section{VAPOR OF AN IONIC FLUID}

As noted above, ion pair molecules dominate the vapor of an ionic fluid such as $\mathrm{NaCl}$ (ref. 21), although there is a substantial population of dimers (quadruple ions such as $\mathrm{Na}_{2} \mathrm{Cl}{ }_{2}$ ). of course, at very low density the ion-pairs dissociate to individual ions, and at higher densities there continue to be significant proportions of single ions and increasing amounts of charged clusters such as $\mathrm{Na}_{2} \mathrm{Cl}^{+}, \mathrm{NaCl}_{2}{ }^{-}$, etc.

For the hard-core model, Gillan (ref. 21) has calculated the approximate fractions of various species up to the neutral hexamer in the saturated vapor and his method is readily extended to the unsaturated vapor. Gillan showed that the saturated vapor of the hard-core, ionic fluid has a much greater density than that given in earlier estimates where the various clusters were not treated explicitly.

For the alkali halide vapors the properties of the ion-pair molecules are well established from spectroscopy (ref. 22). The anharmonic parameters are known as well as the harmonic frequencies and equilibrium distances. Thus, the thermodynamic properties of the monomeric ion-pair vapor can be calculated accurately for temperatures well above those of experimental measurement. Calculations are even more exact for the individual ions. Among larger clusters there are extensive spectroscopic data only for dimer (the neutral $\mathrm{M}_{2} \mathrm{X}_{2}$ cluster) and then only for the harmonic approximation (ref. 22). There are also measurements of the total vapor density. Thus the properties of the alkali halide vapors are quite well known in the temperature range of experimental measurement. With full use of statistical calculations for the monomer, and with guidance from the calculations for the hard-core model, one can make a reasonably good extrapolation to higher temperature (ref. 5). 
The alkali halide vapors follow corresponding states only approximately among themselves. Comparison with the hard-core model on a corresponding states basis shows a much larger difference (ref. 5). The largest discrepancies arise for the ion pair. The real ions are polarizable and the properties of the real ion pairs are very substantially affected by the polarizability. In an ion pair, one ion feels the full electrical field of the other ion. The resulting induced dipole contributes substantial bonding. In larger clusters there are several near neighbors located at different directions and their electrical fields tend to cancel one another at the site of a given ion. This cancellation is essentially complete for the solid and nearly so for the liquid. Thus it was found (ref. 5) that $\mathrm{KCl}$ and $\mathrm{NaCl}$ followed corresponding states quite closely for all properties except the reduced density of the ion pair in the vapor. At $\mathrm{T}_{\mathrm{r}} \cong 0.6$ the density of the $\mathrm{KCl}$ molecule in its vapor is $35 \%$ lower than that of $\mathrm{NaCl}$ in its vapor whereas the densities of $\mathrm{K}_{2} \mathrm{Cl}_{2}$ and $\mathrm{Na}_{2} \mathrm{Cl}_{2}$ follow corresponding states quite accurately. The ion-pair density in the vapor of the hard-core fluid deviates much more; indeed, it is only about one tenth that of $\mathrm{NaCl}$ at a reduced temperature of 0.8 .

\section{$\mathrm{NaCl}$ in steam}

The vapor pressure of pure sodium chloride is to low that one might think it would become appreciably soluble in steam only at very high temperature well above $1000 \mathrm{~K}$. The $\mathrm{NaCl}$ molecular ion pair is strongly hydrated in high-pressure steam, however, at temperatures near the critical point of $\mathrm{H}_{2} \mathrm{O}$. One does not expect the corresponding states principle to be followed with any accuracy for $\mathrm{NaCl}-\mathrm{H}_{2} \mathrm{O}$ in this range. In contrast, it was found (ref. 6) that at higher temperatures and densities, a corresponding states treatment with the water represented as a dielectric was quite successful in fitting the two-component critical curve. But at lower densities the molecular nature of the water must be recognized. This was done (ref. 30) by considering successive hydration equilibria

$$
\operatorname{NaCl}\left(\mathrm{H}_{2} \mathrm{O}\right)_{j-1}+\mathrm{H}_{2} \mathrm{O}=\mathrm{NaCl}\left(\mathrm{H}_{2} \mathrm{O}\right)_{j} .
$$

The corresponding successive hydration equilibria for ions were measured in detail by highpressure mass spectrometry (Kebarle, ref. 31). For $\mathrm{Na}^{+}$the $\Delta \mathrm{H}_{j}$ and $\Delta \mathrm{S}_{j}$ values are known for 6 steps. The $\Delta S_{j}$ values for various steps and for various central ions are all rather near the value $-11.0 \mathrm{R}$. It seems reasonable to assume this same $\Delta S_{j}$ value for the hydration of the ion pair where mass spectrometry cannot yield detailed data.

The enthalpies of hydration were calculated from experimental data on the solubility of solid $\mathrm{NaCl}$ in steam from 350 to $600^{\circ} \mathrm{C}$ and from 30 to 300 bars pressure. Assumptions were made concerning successive $\Delta \mathrm{H}_{j}$ values to reduce the number of independent parameters. Theory suggested a small positive $\Delta C_{p}$ and the value $3 R$ for all steps was selected to give the best fit over a wide range of temperature. Additional details are given in ref. 30 . Certain experimental data show large divergencies, but new measurements of Bischoff et al. (ref. 32) were designed to resolve that problem. It was found that older measurements of olander and Liander (ref. 17) and of Galobardes et a1. (ref. 33) and those above 100 bar of Martynova (ref. 34) were all consistent with the new results and all were used to fit the parameters. As examples of the data, Fig. 6 shows the solubility values along the three-phase line while Fig. 7 shows the data for $450^{\circ} \mathrm{C}$. Measurements of Sourirajan and Kennedy (ref. 35) appear to be unreliable, although they agree at some particular conditions.

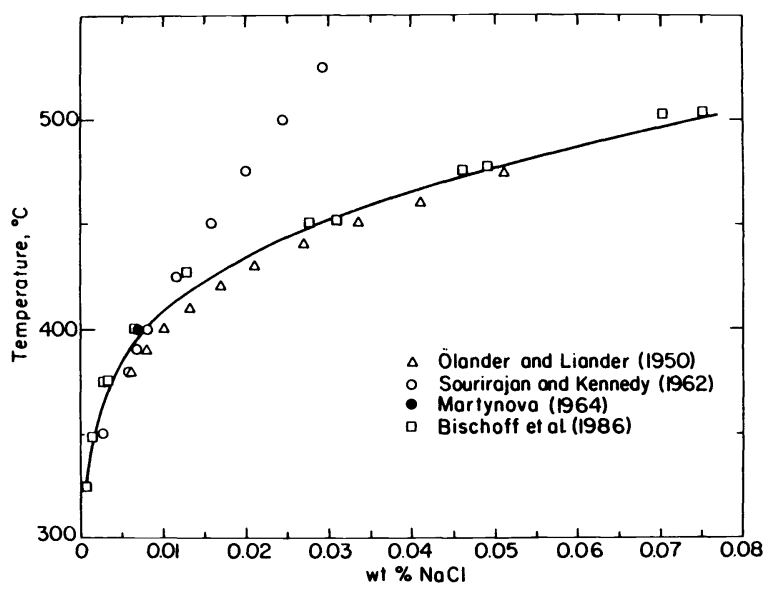

Fig. 6. Vapor phase composition of $\mathrm{NaCl}-\mathrm{H}_{2} \mathrm{O}$ along the three-phase line. The curve is calculated from the model.

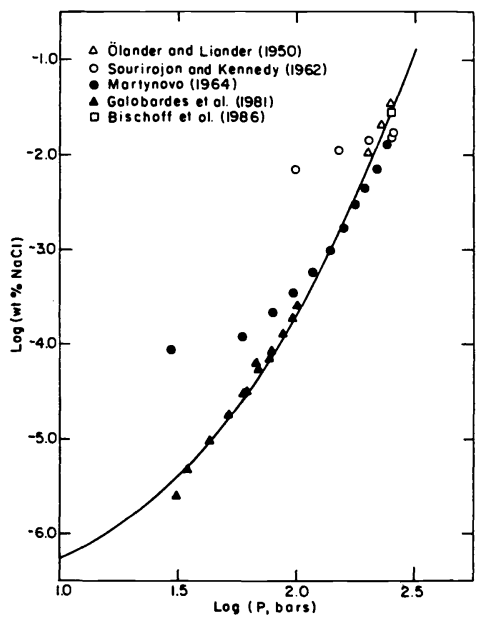

Fig. 7. Vapor phase competition of $\mathrm{NaCl}-\mathrm{H}_{2} \mathrm{O}$ in equilibrium with the solid at $450^{\circ} \mathrm{C}$ as a function of pressure. The curve is calculated from the model. 


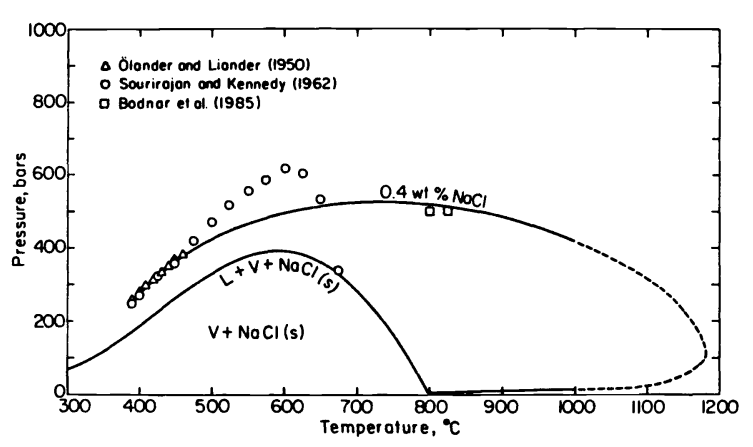

Fig. 8. Isoplethal P-T diagram for the vapor phase composition of $0.4 \mathrm{wt} \% \mathrm{NaCl}$ in $\mathrm{H}_{2} \mathrm{O}$. The curve, which is in the vapor + liquid region, is calculated above $600^{\circ} \mathrm{C}$ and connected smoothly to experimental data at lower temperature. Also shown is the P-T projection of the three-phase line.

Since the degree of hydration decreases with increase in temperature at constant pressure, this model should give valid estimates at still higher temperatures. Figure 8 shows the isoplethal curve calculated for $0.4 \mathrm{wt} \% \mathrm{NaCl}$ which is within this range of validity. Appropriate calculations were made for the fugacity of $\mathrm{NaCl}$ in the liquid phase for these conditions. This curve agrees with very recent measurements of Bodnar et al. (ref. 36) at 800 and $825^{\circ} \mathrm{C}$. In this case again, the measurements of Sourirajan and Kennety (ref. 35) appear to be unreliable. Other predictions of the model for temperatures in the range 800$1100^{\circ} \mathrm{C}$ are of considerable geochemical interest and are discussed in raf. 30.

\section{Acknowledgements}

This work was supported by the Director, Office of Energy Research, Office of Basic Energy Sciences, Division of Chemical Sciences and Division of Engineering and Geosciences of the U. S. Department of Energy under Contract No. DE-AC03-76SF00098.

\section{REFERENCES}

1. K. S. Pitzer, J. Chem. Phys., 7, 583-590 (1940).

2. H. Reiss, S. W. Mayer, and J. L. Katz, J. Chem. Phys., 35, 820-826 (1961).

3. M. Blander, Adv. Chem. Phys., 11, 83-115(1967).

4. H. L. Friedman and B. Larsen, J. Chem. Phys., 70, 92-100 (1979).

5. K. S. Pitzer, Chem. Phys. Lett., 105, 484-489 (1984).

6. K. S. Pitzer, J. Phys. Chem., 88, 2689-2697 (1984).

7. D. Smith-Magowan and R. H. Wood, J. Chem. Thermodyn., 13, 1047-1073 (1981).

8. P. S. Z. Rogers and K. S. Pitzer, J. Phys. Chem., 85, $2886-2895$ (1981); 86, 2110 (1982).

9. H. F. Holmes and R. E. Mesmer, J. Phys. Chem., 87, 1242-1254 (1983).

10. K. S. Pitzer, J. C. Peiper, and R. H. Busey, J. Phys. Chem. Ref. Data, 13, 1-102 (1984).

11. R. C. Phutela and K. S. Pitzer, J. Phys. Chem., 90, 895-901 (1986).

12. K. S. Pitzer and J. J. Kim, J. Am. Chem. Soc., 96, 5701-5707 (1974).

13. C. E. Harvie and J. H. Weare, Geochim. Cosmochim. Acta, 44, 981-997 (1980).

14. M. C. P. de Lima and K. S. Pitzer, J. Soln. Chem., 12, $1 \overline{71}-185$ and 187-199 (1983).

15. M. Buback and E. U. Franck, Ber. Bunsenges. Phys. Chem., 76, 350-354 (1972); 77, 10741079 (1973).

16. K. S. Pitzer, M. C. P. de Lima, and D. R. Schreiber, J. Phys. Chem., 89, 1854-1855 (1985).

17. A. Ölander and H. Liander, Acta Chem. Scand., 4, 1437-1445 (1950).

18. Kh. Khaibullin and M. M. Borisov, Teplofiz. Vyš. Temp., 4, 489-494 (1966) (in English).

19. L. Haar, J. S. Galagher, and G. S. Kel1, NBS-NRC Steam Tables, Hemisphere, Washington (1984).

20. J. L. Bischoff and R. J. Rosenbauer, to be published.

21. M. J. Gillan, Mol. Phys., 49, 421-442 (1983).

22. D. R. Stul1 and H. Prophet, Nat1. Stand. Ref. Data Ser. (U. S. Nat1. Bur. Stand.) NSRDS-NBS 37 (1971).

23. M. C. P. de Lima, D. R. Schreiber, and K. S. Pitzer, to be published.

24. C. W. Davies, Ion Association, Butterworths, London (1962).

25. K. S. Pitzer and J. M. Simonson, J. Am. Chem. Soc., 106, 1973-1977 (1984).

26. D. J. Adams and I. R. McDonald, Physica, 79B, 159-174 (1975).

27. G. N. Papatheodorou, Structure and Thermodynamics of Molten Salts, in Comprehensive Treatise of Electrochemistry, v. 5, Plenum, New York and London (1983).

28. A. D. Kirshenbaum, J. A. Cahil1, P. J. McGonigal, and A. V. Grosse, J. Inorg. Nuc1. Chem., 24, 1287-1296 (1962).

29. S. Biggin and J. E. Enderby, J. Phys. C., 15, L305-309 (1982).

30. K. S. Pitzer and R. T. Pabalan, Geochim. Cosmochim. Acta, 50, in press.

31. P. Kebarle, Annu. Rev. Phys. Chem., 28, 445-476 (1977).

32. J. L. Bischoff, R. J. Rosenbauer, and K. S. Pitzer, Geochim. Cosmochim. Acta, 50, in press.

33. J. F. Galobardes, D. R. Van Hare, and L. B. Rogers, J. Chem. Eng. Data, 26, 363-366 (1981).

34. O. I. Martynova, Russian J. Inorg. Chem., 38, 587-592 (1964).

35. S. Sourirajan and G. C. Kennedy, Am. J. Sci., 260, 115-141 (1962).

36. R. J. Bodnar, C. W. Burnham, and S. M. Sterner, Geochim. Cosmochim. Acta, 49, 1861-1873 (1985). 